\title{
Within-person stability and responsiveness to dietary change of C15:0 and C17:0 concentrations in dry blood spots in the Food4Me Study
}

\author{
V. Albani ${ }^{1}$, M. C. Walsh ${ }^{2}$, M. J. Gibney ${ }^{2}$, J. C. Mathers ${ }^{1}$, A. Adamson ${ }^{1}$, C. Celis-Morales ${ }^{1}$ and \\ L. Brennan ${ }^{1,2}$ on behalf of the Food4Me Study \\ ${ }^{1}$ Human Nutrition Research Centre and Institute of Health and Society, Newcastle University, Newcastle upon Tyne, \\ UK and ${ }^{2}$ Institute of Food and Health, University College Dublin (UCD), Belfield, Dublin, Ireland
}

There is increasing evidence that concentrations in blood of the odd-chain length saturated fatty acids pentadecanoic acid (C15:0) and heptadecanoic acid (C17:0) are biomarkers of dairy intake, and their use in epidemiological studies is growing as more cost-effective methods for collecting biological samples become available $(1,2)$. The aim of this study was to assess the reliability of these biomarkers for estimates of dietary exposure in large epidemiological studies by exploring their stability over time in a population with relatively stable dairy intakes (Control group), and their sensitivity to changes in dairy intake in a population with changing diets (personalised nutrition Intervention group).

Dry blood spot (DBS) samples were collected and dietary intakes from FFQs measured three times over six months (t0, t3 and t6) in both Control and Intervention groups in the Food4Me Study (2). Stability was explored using data from the Control group through Spearman correlation coefficients, ICCs and within person CVs (WCVs) from one-way random effects models of the log-transformed fatty acid concentrations. Sensitivity to changes in diet was explored using differenced regression of log-transformed fatty acid concentrations over daily portions of dairy from the Intervention group.

For the Control group $(\mathrm{N}=760), \mathrm{C} 15: 0$ concentrations showed high correlation over time (ICC: $0 \cdot 62,95 \% \mathrm{CI}: 0 \cdot 57,0 \cdot 68)$, but the ICC for C17:0 was much lower (ICC: $0 \cdot 32,95 \%$ CI: $0 \cdot 28,0 \cdot 46)$. The WCV for C15:0 was $16 \cdot 6 \%(95 \%$ CI: $14 \cdot 9,18 \cdot 3)$ and that for C17:0 was $14 \cdot 6 \%(95 \%$ CI: 13.3,16.0). The highest Spearman correlations were observed for t0-t3 measurements. As with the ICCs, higher values were observed for C15:0 (t0-t3: 0.69, $95 \%$ CI: 0.61, 0.78) than for C17:0 (t0-t3: 0.47, 95\% CI: 0.36, 0.59).

For the Intervention group, there were significant $(p<0.05)$ changes (measured as percentage change in fatty acid concentrations) for C15:0 in DBS and in intakes of total dairy, high-fat dairy, cheese and butter; and for C17:0 in DBS and change in intakes of high-fat dairy and cream.

\begin{tabular}{|c|c|c|c|c|c|}
\hline & \multicolumn{2}{|c|}{ Total dairy } & High-fat dairy & \multicolumn{2}{|c|}{ Low-fat dairy } \\
\hline $\begin{array}{l}\text { C15:0 } \\
\text { C17:0 }\end{array}$ & \multicolumn{2}{|c|}{$\begin{array}{l}1 \cdot 0 *(0.2,1.9) \\
0.1(-0.7,0.9)\end{array}$} & $\begin{array}{r}0 \cdot 3^{*}(0 \cdot 1,0 \cdot 7) \\
0 \cdot 3^{* *}(0 \cdot 1,0 \cdot 5)\end{array}$ & \multicolumn{2}{|c|}{$\begin{array}{l}0.2(-0.4,0.8) \\
0.2(-0.3,0.7)\end{array}$} \\
\hline & Cream & Cheese & Butter & Milk & Yoghurt \\
\hline $\begin{array}{l}\text { C15:0 } \\
\text { C17:0 }\end{array}$ & $\begin{array}{c}0 \cdot 4(-0 \cdot 1,0 \cdot 8) \\
0 \cdot 7^{* *}(0 \cdot 3,1 \cdot 1)\end{array}$ & $\begin{array}{c}1.8^{* *}(0 \cdot 6,3 \cdot 1) \\
0 \cdot 7(-0.4,1 \cdot 8)\end{array}$ & $\begin{array}{c}1 \cdot 0^{* *}(0 \cdot 4,1 \cdot 7) \\
0 \cdot 4(-0 \cdot 3,1 \cdot 1)\end{array}$ & $\begin{array}{c}0 \cdot 4(-1 \cdot 3,2 \cdot 1) \\
-0 \cdot 3(-1 \cdot 7,1 \cdot 2)\end{array}$ & $\begin{array}{r}0.5(-1 \cdot 5,2 \cdot 4) \\
-0 \cdot 8(-2 \cdot 6,1 \cdot 1)\end{array}$ \\
\hline
\end{tabular}

$\%$ change in DBS fatty acid concentration per 1 portion change in dairy intake. $* p<0 \cdot 05, * * p<0 \cdot 01.95 \%$ confidence intervals in parenthesis. All regressions adjusted for total energy intake, age, BMI, smoking status; and intake of alcohol, meat, oily fish, savoury pastries and sweet pastries. Intervention group only $(\mathrm{N}=1247)$.

Results provide evidence of reliability for C15:0 concentrations as measured by stability over time and sensitivity to change in intake of high-fat dairy products. Results for $\mathrm{C} 17: 0$ are less definitive and merit further investigation.

1. Abdullah MMH, Cyr A, Lépine M-C, Labonté M-E, et al. (2015) Br J Nutr 113, 435-44.

2. Celis-Morales C, Livingstone KM, Marsaux CFM, et al. (2014) Genes \& Nutrition 10, 1-13. 